\title{
Superoscillations and Analytic Extension in Schur Analysis
}

\author{
Daniel Alpay ${ }^{1} \cdot$ Fabrizio Colombo $^{2}$ - Irene Sabadini ${ }^{2}$ \\ Received: 5 July 2019 / Revised: 18 July 2020 / Accepted: 25 December 2020 \\ (C) The Author(s) 2021
}

\begin{abstract}
We give applications of the theory of superoscillations to various questions, namely extension of positive definite functions, interpolation of polynomials and also of $R$ functions; we also discuss possible applications to signal theory and prediction theory of stationary stochastic processes. In all cases, we give a constructive procedure, by way of a limiting process, to get the required results.
\end{abstract}

Keywords Superoscillations $\cdot$ Analytic extension $\cdot$ Stationary stochastic processes

Mathematics Subject Classification 30D15 -30A14 · 42A82 - 42A10 $\cdot 60 \mathrm{G} 10$

\section{Introduction}

The notion of superoscillatory functions appears in a series of works of Aharonov, and Berry, see [1-3,19-21], and this mathematical theory has been developed and described in [4-9,11,22-25] and in the monograph [10], and for a historical insight to the function theory see $[12,13,17]$.

The appearance of the superoscillatory phenomenon in quantum mechanics is based on the definition of weak values, see [3]. This value, which is a complex number, comes from a weak measurement of a quantum observable, represented by the self-adjoint operator $A$, involving a pre-selected state $\psi_{0}$ and a post-selected state $\psi_{1}$, and is defined

Communicated by Hans G. Feichtinger.

$凶 \quad$ Fabrizio Colombo

fabrizio.colombo@polimi.it

Daniel Alpay

alpay@chapman.edu

Irene Sabadini

irene.sabadini@polimi.it

1 Faculty of Mathematics, Physics, and Computation, Schmid College of Science and Technology, Chapman University, One University Drive, Orange, CA 92866, USA

2 Politecnico di Milano, Dipartimento di Matematica, Via E. Bonardi, 9, 20133 Milano, Italy 
as

$$
A_{\text {weak }}:=\frac{\left(\psi_{1}, A \psi_{0}\right)}{\left(\psi_{1}, \psi_{0}\right)}=b+i b^{\prime}
$$

Its real part $b$ and its imaginary part $b^{\prime}$ can be interpreted as the shift $b$ and the momentum $b^{\prime}$ of the pointer recording the measurement.

An important feature of the weak measurement is that, in contrast with the strong measurements of von Neumann (given by the expectation value of the operator $A$ )

$$
A_{\text {strong }}:=(\psi, A \psi) \text {, }
$$

the real part $b$ of $A_{\text {weak }}$ can be very large, because $\left(\psi_{1}, \psi_{0}\right)$ can be very small when the states $\psi_{0}$ and $\psi_{1}$ are almost orthogonal. This is what produces the so-called superoscillations.

From the mathematical point of view the archetypical superoscillatory sequence, that first appeared in connection with the theory of weak values, is the sequence of complex valued functions $F_{n}(x, a)$ defined on $\mathbb{R}$ by

$$
F_{n}(x, a)=\left(\cos \left(\frac{x}{n}\right)+i a \sin \left(\frac{x}{n}\right)\right)^{n}=\sum_{k=0}^{n} c_{k}(n, a) e^{i x(1-2 k / n)}
$$

where

$$
c_{k}(n, a)=\left(\begin{array}{l}
n \\
k
\end{array}\right)\left(\frac{1+a}{2}\right)^{n-k}\left(\frac{1-a}{2}\right)^{k},
$$

$a>1$, and $\left(\begin{array}{l}n \\ k\end{array}\right)$ denotes the binomial coefficients. If we fix $x \in \mathbb{R}$, and we let $n$ go to infinity, we immediately obtain that

$$
\lim _{n \rightarrow \infty} F_{n}(x, a)=e^{i a x}, \quad a>1 .
$$

So for $a>1$ the frequency appearing in $e^{i a x}$ is larger then the frequencies $1-2 k / n$ in $e^{i x(1-2 k / n)}$ that are bounded by 1 . From this observation we have the intuitive notion of superoscillatory functions that are, as we will see precisely in the sequel, a special case of super-shift.

To show how superoscillations will be used in this paper, we begin with a question.

Problem 1.1 Assume to know the values of a polynomial p, whose degree is fixed but unknown, on the set

$$
\left\{1-\frac{2 k}{n}, n=1,2, \ldots, k=0, \ldots, n\right\} \subset[-1,1]
$$

(of course, the degree of $p$ is uniquely fixed since we know the values of $p$ on a countable set of points). How can we find the values of $p$ on $\mathbb{R} \backslash[-1,1]$ ?

We cannot use directly the Lagrange interpolating polynomials, since we are not given the degree of $p$. One could define a family of Lagrange interpolating polynomials, of increasing degrees, but convergence is not clear. 
Remark 1.2 This is an extrapolation problem, of the kind considered also in [18,29,30, 34], but here we use different techniques. In fact, we use superoscillations to answer this question. We also note that methods of extrapolation involving Carleman's ideas as developed by Aizenberg, see [14], deserve to be mentioned.

Moreover, considering the coefficients $c_{k}(n, a)$, defined in (1.2), with $a \in \mathbb{R}, n=$ $0,1, \ldots$ and $k=0,1, \ldots, n$, one can prove that the formula

$$
p(a)=\lim _{n \rightarrow \infty} \sum_{k=0}^{n} c_{k}(n, a) p(1-2 k / n), \quad a \in \mathbb{R} \backslash[-1,1]
$$

answers the question.

More generally, consider a function $f$ analytic in an open connected set of the complex plane. By analytic extension, $f$ is uniquely determined by its values on a zero set, but in general no practical formulas seem to be known in general to recover $f$ from this set. In this paper and using the theory of superoscillations we show how one can, in certain cases, give a practical way to recover $f$. As a prelude to the introduction let us state three theorems, whose proofs will be given in the paper. These results can be put under the following common umbrella: When does the limit

$$
\lim _{n \rightarrow \infty} \sum_{k=0}^{n} c_{k}(n, a) f(1-2 k / n), \quad a \in \mathbb{R} \backslash[-1,1]
$$

exists, and what is its significance?

The first result will be of special interest for researchers in function theory; the second result is also in function theory, with possible applications in Schur analysis and in quantum mechanics via the theory of $R$-functions, while the third will be of special interest in the theory of stochastic processes in the case of perfect prediction. In the three results, the stress is on the possibility of an explicit approximation procedure to obtain the solution.

Theorem 1.3 Let $f(z)$ be an entire function of finite exponential type, and assume given $f(t), t \in[-1,1]$. Then, for every real $a \in \mathbb{R} \backslash[-1,1]$ we have

$$
f(a)=\lim _{n \rightarrow \infty} \sum_{k=0}^{n} c_{k}(n, a) f(1-2 k / n)
$$

where the limit is uniform on compact sets on the real line.

Theorem 1.4 Let $d \mu$ be a positive and finite measure on the real line, and assume that $\widehat{\mu}$ has compact support. Let $z=i y_{0}+x, x \in[-1,1]$, with $y_{0}>0$ fixed but arbitrary. Let

$$
\phi(z)=\frac{1}{i} \int_{\mathbb{R}} \frac{d \mu(t)}{t-z} .
$$


Then,

$$
\phi(z)=\lim _{n \rightarrow \infty} \sum_{k=0}^{n} c_{k}(n, x) \phi\left(i y_{0}+(1-2 k / n)\right) .
$$

In other words, we can explicitly recover all values of $\phi$ in the open upper half-plane from the values on the band $[-1,1] \times(0, \infty)$.

In these theorems, analytic extension insures that $f$ and $\phi$ respectively are uniquely determined by their values on a much smaller set, but the point here is to recover the values of the function in an explicit way.

Theorem 1.5 Let $\left(X_{t}\right)_{t \in \mathbb{R}}$ be a zero-mean second order wide sense stationary process in the probability space $\mathbf{L}^{2}(\Omega, \mathcal{C}, P)$, with covariance function $E_{P}\left(X_{t} \overline{X_{s}}\right)=r(t-s)$, and assume that $r$ is entire of finite exponential type. Assume known $r(t)$ for $t \in$ $[-1,1]$. Then, for any $a \in \mathbb{R} \backslash[-1,1]$ we have

$$
X_{a}=\lim _{n \rightarrow \infty} \sum_{k=0}^{n} c_{k}(n, a) X_{(1-2 k / n)}
$$

where the limit in the $\mathbf{L}^{2}(\Omega, \mathcal{C}, P)$ sense.

As explained in the paper, the hypothesis in the theorem imply that we are in the perfect prediction case; equation (1.6) gives a constructive way to find the value $X_{a}$ for any $a \in \mathbb{R} \backslash[-1,1]$.

The purpose of this paper is to discuss links between superoscillations and harmonic analysis, the above theorems being some of the surprising results. The paper consists of five sections besides the introduction. In Sect. 2 we give a short review of the theory of superoscillations. Section 3 contains results on super-shift of entire functions and on interpolation of polynomials. In Sect. 4 we give a result on extension and reconstruction in the setting of positive definite functions on the real line. Connections with the trigonometric moment problems, to the theory of stationary stochastic processes and to signal theory are given in Sect. 6.

\section{A Short Review on Superoscillations}

A superoscillatory function frequently considered in the context of weak measurements is of the type (1.1) where we choose $a>1$ and the coefficients $c_{k}(n, a)$ are given by (1.2). If we fix $x \in \mathbb{R}$ and we let $n$ go to infinity, we obtain that $\lim _{n \rightarrow \infty} F_{n}(x, a)=e^{i a x}$. We point out that in the following we will denote $F_{n}(x, a)$ also by the symbol $F_{n}(x)$ when it is not important to specify the dependence on the parameter $a$. The above fact can be put in precise mathematical terms in the framework of holomorphic entire functions as follows. We recall some notions and results on entire functions. Let $f$ be a non-constant entire function of a complex variable $z$. We define $M_{f}(r)=\max _{|z|=r}|f(z)|$, for $r \geq 0$. The non-negative real number $\rho$ defined by

$$
\rho=\limsup _{r \rightarrow \infty} \frac{\ln \ln M_{f}(r)}{\ln r}
$$


is called the order of $f$. If $\rho$ is finite then $f$ is said to be of finite order and if $\rho=\infty$ the function $f$ is said to be of infinite order. In the case $f$ is of finite order we define the non negative real number

$$
\sigma=\limsup _{r \rightarrow \infty} \frac{\ln M_{f}(r)}{r^{\rho}},
$$

which is called the type of $f$. If $\sigma \in(0, \infty)$ we call $f$ of normal type, while we say that $f$ is of minimal type if $\sigma=0$ and of maximal type if $\sigma=\infty$. The constant functions are said to be of minimal type of order zero.

Definition 2.1 Let $p \geq 1$. We denote by $\mathcal{A}_{p}(\mathbb{C})$ the space of entire functions with either order lower than $p$ or order equal to $p$ and finite type. It consists of entire functions $f$, for which there exist constants $B, C>0$ such that $\forall z \in \mathbb{C}$

$$
|f(z)| \leq C e^{B|z|^{p}}
$$

Let $\left(f_{n}\right)_{n \in \mathbb{N}}, f_{0} \in \mathcal{A}_{p}(\mathbb{C})$. Then $f_{n} \rightarrow f_{0}$ in $\mathcal{A}_{p}(\mathbb{C})$ if there exists some $B>0$ such that

$$
\lim _{n \rightarrow \infty} \sup _{z \in \mathbb{C}}\left|\left(f_{n}(z)-f_{0}(z)\right) e^{-B|z|^{p}}\right|=0
$$

Theorem 2.2 For any $a \in \mathbb{C}$, the sequence $\left\{F_{n}(z, a)\right\}_{n \geq 1}$ converges to $z \mapsto e^{i a z}$ in $\mathcal{A}_{1}(\mathbb{C})$.

Remark 2.3 In particular the convergence in $\mathcal{A}_{1}(\mathbb{C})$ implies the convergence on the compact sets of $\mathbb{C}$.

Lemma 2.4 Let $a \in \mathbb{R}$ and let $F_{n}(z, a)$ be as in definition (1.1) for $z \in \mathbb{C}$. Then, for all $p \in \mathbb{N}$

$$
\lim _{n \rightarrow \infty} \partial_{z}^{(p)} F_{n}(z, a)=\partial_{z}^{(p)} e^{i a z}
$$

uniformly on every compact set $K$ in $\mathbb{C}$.

The precise definition of superoscillatory functions is as follows.

Definition 2.5 (Generalized Fourier sequence). We call generalized Fourier sequence a sequence of the form

$$
Y_{n}(x, a):=\sum_{j=0}^{n} C_{j}(n, a) e^{i k_{j}(n) x}
$$

where $a \in \mathbb{R}, C_{j}(n, a)$ and $k_{j}(n)$ are real valued functions of the variables $n, a$ and $n$, respectively.

The sequence of partial sums of a Fourier expansion is a particular case of this notion with $C_{j}(n, a)=C_{j} \in \mathbb{R}$ and $k_{j}(n)=k_{j} \in \mathbb{R}$. 
Definition 2.6 (Superoscillating sequence). Let $a \in \mathbb{R}$. A generalized Fourier sequence

$$
Y_{n}(x, a)=\sum_{j=0}^{n} C_{j}(n, a) e^{i k_{j}(n) x}
$$

is said to be a superoscillating sequence if:

- $\left|k_{j}(n)\right| \leq 1$;

- there exists a compact subset of $\mathbb{R}$, which will be called a superoscillation set, on which $Y_{n}$ converges uniformly to $e^{i g(a) x}$ where $g$ is a continuous real value function such that $|g(a)|>1$.

The classical Fourier expansion is obviously not a superoscillating sequence since its frequencies are not, in general, bounded. It is possible to construct a large class of superoscillatory function using continuity theorems of suitable convolution operators acting on spaces of entire functions. For example, consider the function

$$
\psi_{n}(x, t)=\sum_{j=0}^{n} c_{k}(n, a) e^{i x(1-2 k / n)^{m}}, \quad m \in \mathbb{N},
$$

where $c_{k}(n, a)$ are defined in (1.2) taking the limit we have

$$
\lim _{n \rightarrow \infty} \psi_{n}(x, t)=e^{i a^{m} x}
$$

More general we can consider holomorphic functions $g$ such that under suitable conditions the sequence

$$
\phi_{n}(x, t)=\sum_{k=0}^{n} c_{k}(n, a) e^{i x g(1-2 k / n)}
$$

where $c_{k}(n, a)$ are defined in (1.2) is superoscillatory and

$$
\lim _{n \rightarrow \infty} \phi_{n}(x, t)=e^{i g(a) x} .
$$

\section{Super-Shift of Polynomials and of Entire Functions}

In this section we show how to answer the first question in the Introduction, namely, how to find the values of a polynomial $p$ of fixed, but unknown, degree, knowing its values in $[-1,1]$. To this end, we consider the points $1-2 k / n \subseteq[-1,1]$ and the sequence

$$
n \mapsto \sum_{k=0}^{n} c_{k}(n, a) p(1-2 k / n)
$$

We have: 
Theorem 3.1 Let $p$ be a polynomial. Then,

$$
\lim _{n \rightarrow \infty} \sum_{k=0}^{n} c_{k}(n, a) p(1-2 k / n)=p(a) \quad a \in \mathbb{R} \backslash[-1,1]
$$

Proof We first prove

$$
\lim _{n \rightarrow \infty} \sum_{k=0}^{n} c_{k}(n, a) \frac{k^{u}}{n^{u}}=\left(\frac{1-a}{2}\right)^{u}, \quad u=0,1, \ldots
$$

and proceed by induction on the degree $u$ of $p$. For degree 0 , the result is true since $\sum_{k=0}^{n} c_{k}(n, a)=1$. Assume the result true at degree $u-1$. We can write

$$
\begin{aligned}
\sum_{k=1}^{n} c_{k}(n, a) \frac{k^{u}}{n^{u}} & =\sum_{k=1}^{n} \frac{k}{n}\left(\begin{array}{l}
n \\
k
\end{array}\right)\left(\frac{1+a}{2}\right)^{n-k}\left(\frac{1-a}{2}\right)^{k} \frac{k^{u-1}}{n^{u-1}} \\
& =\left(\frac{1-a}{2}\right)\left(\sum_{k=1}^{n}\left(\begin{array}{l}
n-1 \\
k-1
\end{array}\right)\left(\frac{1+a}{2}\right)^{n-k}\left(\frac{1-a}{2}\right)^{k-1} \frac{k^{u-1}}{n^{u-1}}\right) \\
& =\left(\frac{1-a}{2}\right) \frac{(n-1)^{u}}{n^{u}}\left(\sum_{k=1}^{n}\left(\begin{array}{l}
n-1 \\
k-1
\end{array}\right)\left(\frac{1+a}{2}\right)^{n-k}\left(\frac{1-a}{2}\right)^{k-1} \frac{k^{u-1}}{(n-1)^{u-1}}\right)
\end{aligned}
$$

and so

$$
\begin{aligned}
\lim _{n \rightarrow \infty} \sum_{k=0}^{n} c_{k}(n, a) \frac{k^{u}}{n^{u}} & =\frac{1-a}{2} \lim _{n \rightarrow \infty} \sum_{k=0}^{n-1} c_{k}(n-1, a) \frac{k^{u-1}}{(n-1)^{u-1}} \\
& =\frac{1-a}{2}\left(\frac{1-a}{2}\right)^{u-1} \\
& =\left(\frac{1-a}{2}\right)^{u} .
\end{aligned}
$$

We now prove (3.1). It is enough to prove the result for monomials. We have for $u=0,1, \ldots$, and using (3.2) to get to the last line,

$$
\begin{aligned}
\lim _{n \rightarrow \infty} \sum_{k=0}^{n} c_{k}(n, a)\left(1-\frac{2 k}{n}\right)^{u} & =\lim _{n \rightarrow \infty} \sum_{k=0}^{n} c_{k}(n, a)\left(\sum_{r=0}^{u}\left(\begin{array}{l}
u \\
r
\end{array}\right)\left(\frac{2 k}{n}\right)^{r}(-1)^{r}\right) \\
& =\sum_{r=0}^{u} 2^{r}(-1)^{r}\left(\begin{array}{l}
u \\
r
\end{array}\right)\left(\lim _{n \rightarrow \infty} \sum_{k=0}^{n} c_{k}(n, a)\left(\frac{k}{n}\right)^{r}\right) \\
& =\sum_{r=0}^{u} 2^{r}(-1)^{r}\left(\begin{array}{l}
u \\
r
\end{array}\right)\left(\frac{1-a}{2}\right)^{r}
\end{aligned}
$$




$$
\begin{aligned}
& =\sum_{r=0}^{u}\left(\begin{array}{l}
u \\
r
\end{array}\right)(a-1)^{r} \\
& =a^{u} .
\end{aligned}
$$

Formula (3.1) allows to reconstruct a polynomial of any degree from its values at the points $1-\frac{2 k}{n}, n=1,2, \ldots$, and $k=0,1, \ldots, n$.

The above observation and the definition of superoscillatory functions naturally lead the definition of super-shift.

Definition 3.2 (Super-shift). Let $\mathcal{I} \subseteq \mathbb{R}$ be an interval with $[-1,1] \subset \mathcal{I}, \Omega \subseteq \mathbb{R}^{d}$ be a domain and let $\varphi: \mathcal{I} \times[0, T] \times \Omega \rightarrow \mathbb{R}, T>0$ be a continuous function on $\mathcal{I}$. If $\varphi_{\lambda}(t, x):=\varphi(\lambda, t, x)$, we can consider a sequence of points $\left\{\lambda_{k, n}\right\}$ in $[-1,1]$ for $n=0, \ldots,+\infty$, and a sequence of complex numbers $\left\{c_{k}(n)\right\}$, and define the functions

$$
\psi_{n}(t, x)=\sum_{k=0}^{n} c_{k}(n) \varphi_{\lambda_{k, n}}(t, x) .
$$

If

$$
\lim _{n \rightarrow \infty} \psi_{n}(t, x)=\varphi_{a}(t, x)
$$

for some $a \in \mathcal{I}$, we say that the function $\lambda \rightarrow \varphi_{\lambda}(t, x)$, for $t$ and $x$ fixed, admits a super-shift in $\lambda$.

The previous result generalizes to entire functions:

Theorem 3.3 Let $f \in \mathcal{A}_{1}(\mathbb{C})$, i.e. let $f$ be an entire function such that there exist $C_{f}>0, b>0$ for which

$$
\left|\alpha_{u}\right| \leq C_{f} \frac{b^{u}}{u !}
$$

Then we have

$$
f(a)=\lim _{n \rightarrow \infty} \sum_{k=0}^{n} c_{k}(n, a) f(1-2 k / n) .
$$

Proof We introduce the auxiliary complex variable $\xi$ so that

$$
D_{\xi}^{r} e^{i \beta \xi}=(i \beta)^{r} e^{i \beta \xi}
$$

so

$$
\beta^{r}=\left.\frac{1}{i^{r}} D_{\xi}^{r} e^{i \beta \xi}\right|_{\xi=0} .
$$


We have the following chain of equalities:

$$
\begin{aligned}
\sum_{k=0}^{n} c_{k}(n, a) f(1-2 k / n) & =\sum_{u=0}^{\infty} \alpha_{u} \sum_{k=0}^{n} c_{k}(n, a)(1-2 k / n)^{u} \\
& =\left.\sum_{u=0}^{\infty} \alpha_{u} \sum_{k=0}^{n} c_{k}(n, a) \frac{1}{i^{u}} D_{\xi}^{u} e^{i(1-2 k / n) \xi}\right|_{\xi=0} \\
& =\left.\sum_{u=0}^{\infty} \alpha_{u} \frac{1}{i^{u}} D_{\xi}^{u} \sum_{k=0}^{n} c_{k}(n, a) e^{i(1-2 k / n) \xi}\right|_{\xi=0} \\
& =\left.\sum_{u=0}^{\infty} \alpha_{u} \frac{1}{i^{u}} D_{\xi}^{u} F_{n}(\xi, a)\right|_{\xi=0}
\end{aligned}
$$

Now we consider the operator

$$
U\left(D_{\xi}\right):=\sum_{u=0}^{\infty} \alpha_{u} \frac{1}{i^{u}} D_{\xi}^{u}
$$

and since the coefficients $\alpha_{u}$ satisfy the condition (3.4) we have by Theorem 2.3 in [17] (see also [16] for more details) that it acts continuously on the functions in $\mathcal{A}_{1}(\mathbb{C})$. So we get

$$
\begin{aligned}
\lim _{n \rightarrow \infty} \sum_{k=0}^{n} c_{k}(n, a) f(1-2 k / n)= & \left.\lim _{n \rightarrow \infty} U\left(D_{\xi}\right) F_{n}(\xi, a)\right|_{\xi=0} \\
& =\left.U\left(D_{\xi}\right) \lim _{n \rightarrow \infty} F_{n}(\xi, a)\right|_{\xi=0} \\
& =\left.U\left(D_{\xi}\right) e^{i a \xi}\right|_{\xi=0} \\
& =\sum_{u=0}^{\infty} \alpha_{u} a^{u}=f(a),
\end{aligned}
$$

and this concludes the proof.

Remark 3.4 The proof of Theorem 3.3 suggests a second proof of Theorem 3.1. In fact, we have

$$
\sum_{k=0}^{n} c_{k}(n, a) p(1-2 k / n)=\sum_{u=0}^{N} \alpha_{u} \sum_{k=0}^{n} c_{k}(n, a)(1-2 k / n)^{u}
$$


Using (3.5) we can write

$$
\begin{aligned}
\sum_{k=0}^{n} c_{k}(n, a) P_{N}(1-2 k / n) & =\left.\sum_{u=0}^{N} \alpha_{u} \frac{1}{i^{u}} D_{\xi}^{u} \sum_{k=0}^{n} c_{k}(n, a) e^{i(1-2 k / n) \xi}\right|_{\xi=0} \\
& =\left.\sum_{u=0}^{N} \alpha_{u} \frac{1}{i^{u}} D_{\xi}^{u} F_{n}(\xi, a)\right|_{\xi=0} .
\end{aligned}
$$

Now we observe that

$$
\begin{aligned}
\left.\lim _{n \rightarrow \infty} \sum_{u=0}^{N} \alpha_{u} \frac{1}{i^{u}} D_{\xi}^{u} F_{n}(\xi, a)\right|_{\xi=0} & =\left.\sum_{u=0}^{N} \alpha_{u} \frac{1}{i^{u}} D_{\xi}^{u} \lim _{n \rightarrow \infty} F_{n}(\xi, a)\right|_{\xi=0} \\
& =\left.\sum_{u=0}^{N} \alpha_{u} \frac{1}{i^{u}} D_{\xi}^{u} e^{i a \xi}\right|_{\xi=0}=p(a) .
\end{aligned}
$$

and this concludes the proof.

\section{Extension}

Given a function $D(a), a \in[-1,1]$ positive definite in $[-1 / 2,1 / 2]$, the theory of the description of all its positive definite extensions to the real line has a long history, see [33] and the references therein. The problem can be formulated as follows:

Problem 4.1 Find necessary and sufficient conditions on a given a function $D(a), a \in$ $[-1,1]$ for the existence of a positive measure $\mu$ with compact support $K$ such that

$$
\int_{K} e^{i a t} d \mu(t)=D(a), \quad a \in[-1,1] .
$$

Here we study this problem using the theory of superoscillations. We first give necessary conditions:

Lemma 4.2 Assume that Problem 4.1 has a solution. Then the function D is necessarily continuous in $[-1,1]$, satisfies $D(-a)=\overline{D(a)}$, and the kernel $k(a, b)=D(a-b)$ is positive definite for $a, b \in(-1 / 2,1 / 2)$. Finally, $D$ is the restriction to $[-1,1]$ of $a$ uniquely defined entire function of finite exponential type and the limit

$$
\lim _{n \rightarrow \infty} \sum_{k=0}^{n} c_{k}(n, a) D(1-2 k / n)
$$

exists for every real number a. 
Proof Let $\left(a_{n}\right)_{n \in \mathbb{N}}$ be a sequence of real points with limit $a$. Since the support $K$ is a compact set in $\mathbb{R}$, by dominated convergence can interchange limit with respect to $a$ and integration and write

$$
\lim _{n \rightarrow \infty} D\left(a_{n}\right)=\lim _{n \rightarrow \infty} \int_{K} e^{i a_{n} t} d \mu(t)=\int_{K} \lim _{n \rightarrow \infty} e^{i a_{n} t} d \mu(t)=D(a) .
$$

Interchanging complex adjoint and integration gives $D(-a)=\overline{D(a)}$. The positivity claim follows from

$$
D(a-b)=\int_{K} e^{i a t} e^{-i b t} d \mu(t), \quad a, b \in(-1 / 2,1 / 2) .
$$

$D(a)$ is the restriction to $[-1,1]$ of an entire function, and so has a unique extension which is entire. Since the sequence of functions $F_{n}(t, a)$ converges uniformly on compact sets to $e^{i a t}$ we can write

$$
\int_{K} e^{i a t} d \mu(t)=\lim _{n \rightarrow \infty} \sum_{k=0}^{n} c_{k}(n, a) D(1-2 k / n)
$$

which is (4.2).

Formula (4.3) expresses that the function $\int_{0}^{2 \pi} e^{i a t} e^{-i b t} d \mu(t)$ is a positive definite extension of $D(a-b)$ to the whole real line.

Theorem 4.3 Let $D$ be continuous on $[-1,1]$, such that the kernel $k(a, b)=D(a-b)$ is positive definite on $[-1 / 2,1 / 2]$, and $D$ is the restriction to $[-1,1]$ of an entire function of finite exponential type. Then the limit (4.2) exists, and defines a positive definite function. This function is the only positive extension of $D$.

Proof The function $D$ has positive definite extensions to $\mathbb{R}$, see [33], and by Bochner's theorem (see [35, p. 333]) we can write any of these extensions in the form

$$
\int_{\mathbb{R}} e^{i a t} d \sigma(t)
$$

The measure $d \sigma$ defines a distribution on the space of Schwartz functions, and its Fourier transform is an entire function of finite exponential type. By the Paley-Wiener theorem, the support of $d \sigma$ is finite, say $K$, and so

$$
\sum_{k=0}^{n} c_{k}(n, a) D(1-2 k / n)=\int_{K}\left(\sum_{k=0}^{n} c_{k}(n, a) e^{i(1-2 k / n) t}\right) d \sigma(t) .
$$


Since $K$ is compact, one proceeds as in Lemma 4.2, interchange the limit and the integral, and write

$$
\begin{aligned}
\lim _{n \rightarrow \infty} \int_{K}\left(\sum_{k=0}^{n} c_{k}(n, a) e^{i(1-2 k / n) t}\right) d \sigma(t) & =\int_{K} \lim _{n \rightarrow \infty}\left(\sum_{k=0}^{n} c_{k}(n, a) e^{i(1-2 k / n) t}\right) d \sigma(t) \\
& =\int_{K} e^{i a t} d \sigma(t) .
\end{aligned}
$$

The function $f(a)=\int_{K} e^{i a t} d \sigma(t)$ is positive definite on the real line, and by analyticity is the only entire positive extension of $D$. It is in fact the only positive extension of $D$, since by the above construction, two such extensions $D_{1}$ and $D_{2}$ would be such that

$$
D(a)=D_{1}(a)=D_{2}(a)=\int_{K_{1}} e^{i a t} d \sigma_{1}(t)=\int_{K_{2}} e^{i a t} d \sigma_{2}(t), \quad a \in[-1 / 2,1 / 2]
$$

where $\sigma_{1}$ and $\sigma_{2}$ are positive finite measures with respective supports the compact sets $K_{1}$ and $K_{2}$.

When the interval $[-\pi, \pi]$ is chosen in (4.1) connections with the trigonometric moment problem (see e.g. [15]) can be made, but other choices of compact sets are also of special interest.

Other classes of measures can be considered, which include the compact support case.

Definition 4.4 We denote by $\mathscr{M}_{1}$ the set of positive finite measures on $\mathbb{R}$ such that

$$
\int_{\mathbb{R}} e^{C|x|} d \sigma(x)<\infty, \quad \forall C \geq 0
$$

For instance measures with compact support or measures such as $e^{-x^{2}} d x$ belong to $\mathscr{M}_{1}$. We note also that the class $\mathscr{M}_{1}$ is related to the discussion in [35, p. 335].

Problem 4.5 Given a function $D(a), a \in[-1,1]$ find a necessary and sufficient condition for a positive measure $\sigma \in \mathscr{M}_{1}$ such that

$$
\int_{\mathbb{R}} e^{i a t} d \sigma(t)=D(a), \quad a \in[-1,1]
$$

to exist, and give a formula for $\sigma$.

By (2.2) for every $a \in \mathbb{R}$ there exist $B_{a}$ and $N_{a}$ (which depend on $a$ ) such that

$$
n \geq N_{a} \Longrightarrow \sup _{z \in \mathbb{C}}\left|\left(F_{n}(z, a)-e^{i a z}\right) e^{-B_{a}|z|}\right| \leq 1
$$

and thus for $n \geq N_{a}$, and restricting $z$ to be real,

$$
\left|F_{n}(x, a)\right| \leq 1+e^{B_{a}|x|}, \quad \forall x \in \mathbb{R} .
$$


The dominated convergence theorem allows then to conclude that, for any given $a \in \mathbb{R}$

$$
\begin{aligned}
\lim _{n \rightarrow} \int_{\mathbb{R}}\left|F_{n}(x, a)-e^{i a x}\right| d \sigma(x) & =\lim _{n \rightarrow} \int_{\mathbb{R}}\left|F_{n}(x, a)-e^{i a x}\right| e^{-B_{a}|x|} e^{B_{a}|x|} d \sigma(x) \\
& =0
\end{aligned}
$$

More generally, for $p \geq 1$, one can introduce the class $\mathscr{M}_{p}$ the set of positive finite measures on $\mathbb{R}$ such that

$$
\int_{\mathbb{R}} e^{C|x|^{p}} d \sigma(x)<\infty, \quad \forall C \geq 0
$$

which can be of independent interest, although we do not use them in this paper. We note that $\mathscr{M}_{p} \subset \mathscr{M}_{q} \subset \mathscr{M}_{1}$, for $p \geq q \geq 1$.

\section{$5 \boldsymbol{R}$-Functions and Interpolation}

Let $d \mu$ be a positive and finite measure on the real line, and let

$$
\phi(z)=\frac{1}{i} \int_{\mathbb{R}} \frac{d \mu(t)}{t-z} .
$$

Then for $z, w \in \mathbb{C} \backslash \mathbb{R}$,

$$
\frac{\phi(z)+\overline{\phi(w)}}{-i(z-\bar{w})}=\int_{\mathbb{R}} \frac{d \mu(t)}{(t-z)(t-\bar{w})}
$$

so that $i \phi$ is a Nevanlinna function (also known as $R$-function). For $\operatorname{Im} z>0$ we have

$$
\begin{aligned}
\phi(z) & =\frac{1}{i} \int_{\mathbb{R}} \frac{d \mu(t)}{t-z} \\
& =\int_{\mathbb{R}} d \mu(t) \int_{0}^{\infty} e^{-i(t-z) u} d u \\
& =\int_{0}^{\infty} e^{i z u}\left(\int_{\mathbb{R}} e^{-i t u} d \mu(t)\right) d u \\
& =\int_{0}^{\infty} e^{i z u} \widehat{\mu}(u) d u
\end{aligned}
$$

where we denote by $\widehat{\mu}(u)=\int_{\mathbb{R}} e^{-i t u} d \mu(t)$ the Fourier transform of the measure $\mu$.

Theorem 5.1 When $\widehat{\mu}$ has compact support, one can recover $\phi\left(i y_{0}+x\right), x \in \mathbb{R}$, from $\phi(z), z=i y_{0}+x, x \in[-1,1]$, with $y_{0}>0$ fixed but arbitrary, via the limit

$$
\phi(z)=\lim _{n \rightarrow \infty} \sum_{k=0}^{n} c_{k}(n, x) \phi\left((1-2 k / n)+i y_{0}\right) .
$$


Proof Let $z=i y_{0}+x$, with $x \in[-1,1]$. We have

$$
\begin{aligned}
\phi(z) & =\int_{0}^{\infty} e^{i u x} e^{-y_{0} u \widehat{\mu}(u) d u} \\
& =\int_{0}^{\infty} \lim _{n \rightarrow \infty}\left(\sum_{k=0}^{n} c_{k}(n, x) e^{i(1-2 k / n) u}\right) e^{-y_{0} u \widehat{\mu}(u) d u} \\
& =\lim _{n \rightarrow \infty} \int_{0}^{\infty}\left(\sum_{k=0}^{n} c_{k}(n, x) e^{i(1-2 k / n) u}\right) e^{-y_{0} u \widehat{\mu}(u) d u} \\
& =\lim _{n \rightarrow \infty} \sum_{k=0}^{n} \int_{0}^{\infty} c_{k}(n, x) e^{i\left((1-2 k / n)+i y_{0}\right) u} \widehat{\mu}(u) d u \\
& =\lim _{n \rightarrow \infty} \sum_{k=0}^{n} c_{k}(n, x) \phi\left((1-2 k / n)+i y_{0}\right) .
\end{aligned}
$$

The above proof still holds if $\widehat{\mu}$ has the following property: There exists $y_{0}>0$ such that $\int_{0}^{\infty} e^{-y_{0} u} \widehat{\mu}(u) d u$ converges absolutely. Using the dominated convergence theorem, one can then recover explicitly the values of $\phi$ for $(x, y) \in[-1,1] \times\left(0, y_{0}\right)$ via (5.2).

\section{Some Applications}

In this section we discuss some possible applications of the ideas and techniques illustrated in this paper to some other settings.

We start by discussing links of Sect. 4 with the trigonometric moment problem. Let us consider $D$ as in Sect. 4, and let us denote still by $D$ its entire extension. Then we have:

$$
|D(\lambda)| \leq C e^{C_{2}|\operatorname{Im} \lambda|}, \quad \lambda \in \mathbb{C},
$$

where $C_{2} \geq 0$. By Paley-Wiener theorem, the support $K$ of the distribution $d \sigma$ is inside the interval $\left[-C_{2}, C_{2}\right]$. We now assume that $C_{2} \leq \pi$.

Theorem 6.1 Assume that $C_{2} \leq \pi$ in (6.1). There is a unique measure $d \mu$ satisfying (4.1) with $K=[-\pi, \pi]$ and we have:

$$
\begin{gathered}
\int_{-\pi}^{\pi} \frac{e^{i t}+z}{e^{i t}-z} d \mu(t)=D(0)+2 \sum_{m=1}^{\infty} z^{m}\left(\lim _{n \rightarrow \infty}\left(\sum_{k=0}^{n} c_{k}(n, m) D(-(1-2 k / n))\right)\right), \\
|z|<1 .
\end{gathered}
$$

Proof Let

$$
R(m)=\lim _{n \rightarrow \infty} \sum_{k=0}^{n} c_{k}(n, m) D(1-2 k / n), \quad m=2,3, \ldots
$$


Then the Toeplitz matrices

$$
T_{M}=(R(u-v))_{u, v=0, \ldots M}
$$

are all non-negative since the function (4.2) is positive definite on the real line.

The second claim is a standard result from the trigonometric moment problem. Write $R(m)=\int_{-\pi}^{\pi} e^{i m u} d \mu(u)$. Then, on the one hand

$$
\varphi(z)=\int_{-\pi}^{\pi} \frac{e^{i t}+z}{e^{i t}-z} d \mu(t)
$$

and on the other hand we have:

$$
\begin{aligned}
\varphi(z) & =r(0)+2 \sum_{m=1}^{\infty} R(-m) z^{m} \\
& =D(0)+2 \sum_{m=0}^{\infty} z^{m}\left(\lim _{n \rightarrow \infty}\left(\sum_{k=0}^{n} c_{k}(n, m) D(-(1-2 k / n))\right)\right) .
\end{aligned}
$$

Section 4 also links with stationary stochastic processes. Let $(\Omega, \mathcal{C}, P)$ be a probability space, that is, a measure space, endowed with a sigma-algebra $\mathcal{C}$ and a positive measure $P$ (called probability measure) satisfying $P(\Omega)=1$. Functions from $(\Omega, \mathcal{C}, P)$ into $\mathbb{C}$ are called random variables. A function $X$ from some set $T$ into $\mathbf{L}^{2}(\Omega, \mathcal{C}, P)$ is called a second order probability process. We denote by $E_{P}$ the mathematical expectation:

$$
E_{P}(X)=\int_{\Omega} X(\omega) d P(\omega),
$$

and recall that the covariance function of $X$ is the positive definite function defined by

$$
K_{X}(t, s)=E_{P}\left(X_{t} \overline{X_{s}}\right) .
$$

We will assume here $T=\mathbb{R}$ or a sub-interval of $\mathbb{R}$, or $T=\mathbb{Z}$, or a subset of $\mathbb{Z}$. The process is called wide-sense stationary (we will say stationary in this section) if function $(t, s) \mapsto E_{P}\left(X_{t} \overline{X_{s}}\right)$ depends only of the difference $t-s$ then

$$
E_{P}\left(X_{t} \overline{X_{S}}\right)=r(t-s)
$$

where $r$ is called the the covariance function.

In the case $T=\mathbb{R}$, assuming that $r$ is continuous at the origin, Bochner's theorem insures that

$$
r(t)=\int_{\mathbb{R}} e^{i t u} d \sigma(u)
$$


where $\sigma$ is a positive measure, see [35], and it follows that we have the isomorphism $X_{t} \Longleftrightarrow e^{i t u}$, called the trigonometric isomorphism, from $\mathbf{L}^{2}(\Omega, \mathcal{C}, P)$ into $\mathbf{L}^{2}(\mathbb{R}, \mathcal{B}, d \sigma)$. Let $m(u)$ denote the absolutely continuous part in the decomposition of $\sigma$ as a sum of a measure absolutely continuous with Lebesgue measure and a measure orthogonal to the Lebesgue measure. We assume that $d \sigma$ has bounded support. It follows in particular that

$$
\int_{\mathbb{R}} \frac{\ln m(u)}{1+u^{2}} d u=-\infty .
$$

By Szegö's alternative theorem, the closed linear span of the exponentials is equal to $\mathbf{L}^{2}(\mathbb{R}, \mathcal{B}, d \sigma)$. We are in the case of perfect prediction. The trigonometric isomorphism allows to interpret formula (4.4) as an explicit way of solving the extrapolation problem for stationary processes in the case of perfect prediction, namely we have:

Theorem 6.2 Let $\left(X_{t}\right)_{t \in \mathbb{R}}$ be a zero-mean second order wide sense stationary process in the probability space $\mathbf{L}^{2}(\Omega, \mathcal{C}, P)$, with covariance function $E_{P}\left(X_{t} \overline{X_{S}}\right)=r(t-s)$, and assume that $r$ is entire of finite exponential type. Assume known $r(t)$ for $t \in$ $[-1,1]$. Then, for any $a \in \mathbb{R} \backslash[-1,1]$ we have

$$
X_{a}=\lim _{n \rightarrow \infty} \sum_{k=0}^{n} c_{k}(n, a) X_{(1-2 k / n)}
$$

where the limit is in the $\mathbf{L}^{2}(\Omega, \mathcal{C}, P)$ sense.

Stationary increments processes form an important class of stochastic processes, whose covariance functions were studied in particular by Krein, Schoenberg and von Neumann; see $[31,32,36]$. These are functions of the form:

$$
\int_{\mathbb{R}} \frac{e^{i t u}-1}{u} \frac{e^{-i s u}-1}{u} d \sigma(u)=r(t)+\overline{r(s)}-r(t-s) .
$$

Assuming that $d \sigma$ has compact support, and of the form

$$
\sigma(u)=u d \mu(u)
$$

we can take

$$
r(t)=\int_{\mathbb{R}}\left(1-e^{i t u}\right) d \mu(u)
$$

and we have

$$
\begin{aligned}
r(t) & =\mu(\mathbb{R})-\lim _{n \rightarrow \infty} \sum_{k=0}^{n} c_{k}(n, t)(\mu(\mathbb{R})-r(1-2 k / n)) \\
& =\lim _{n \rightarrow \infty} \sum_{k=0}^{n} c_{k}(n, t) r(1-2 k / n)
\end{aligned}
$$

since $\sum_{k=0}^{n} c_{k}(n, t)=1$. 
Remark 6.3 de Branges spaces in this context can be defined following a standard argument. Let $d \mu$ be a positive measure on the real line such that $\int_{\mathbb{R}} \frac{d \mu(t)}{t^{2}+1}<\infty$. For a given $T>0$, the closed linear span $H_{T}$ of the functions $u \mapsto \frac{e^{i t u}-1}{u},|t| \leq$ $T$, plays an important role in prediction theory; when $H_{T} \neq \mathbf{L}^{2}(\mathbb{R}, \mu)$, it has a reproducing kernel of a special form. These spaces were first introduced and studied by de Branges; see [27], and their application to prediction theory was developed by Dym and McKean; see [28,29]. However, this theory will not help here, since we are in the perfect prediction case. The map which to $f \in \mathbf{L}^{2}(\mathbb{R}, \mathcal{B}, d \sigma)$ associates the function

$$
F_{f}(t)=\int_{\mathbb{R}} e^{i t u} f(u) d \sigma(u)
$$

is an isomorphism between $\mathbf{L}^{2}(\mathbb{R}, \mathcal{B}, d \sigma)$ and the reproducing kernel Hilbert space with reproducing kernel

$$
\int_{\mathbb{R}} e^{i(t-s) u} d \sigma(u)
$$

and one can recover the values of $F_{f}(t)$ in $\mathbb{R}$ from its values in $[-1,1]$ as

$$
F_{f}(t)=\lim _{n \rightarrow \infty} \sum_{k=0}^{n} c_{k}(t, n) F_{f}(1-2 k / n) .
$$

To quote [28, p. 302], perfect prediction of the future on the basis of the whole past is possible precisely in the non-Hardy space.

Finally, it is well known that the theory of superoscillations has applications to signal theory and in the literature there are plenty of results in this direction, starting from the early paper by Berry, see [19].

Here we consider a signal $s(t)$ with band-limited spectrum, so that its Fourier transform (i.e. its spectrum) $\widehat{s}(\omega)=\int_{\mathbb{R}} e^{-i \omega t} s(t) d t$ has a compact support, which we will assume symmetric and denote as $[-M, M]$. We can write

$$
s(t)=\frac{1}{2 \pi} \int_{[-M, M]} e^{i \omega t} \widehat{s}(\omega) d w,
$$

and in particular $s$ is (the restriction to the real line) of an entire function of finite exponential type. By analytic extension it can, in principle, be recovered knowing its values on a zero set, but, as we mentioned above, no practical formula seems to be known in general. In another direction, Shannon's sampling theorem asserts that

$$
s(t)=\sum_{n \in \mathbb{Z}} s\left(\frac{n \pi}{M}\right) \frac{\sin (M t-n \pi)}{M t-n \pi},
$$

and

$$
\int_{\mathbb{R}}|s(t)|^{2} d t=\frac{\pi}{M} \sum_{n \in \mathbb{Z}}\left|s\left(\frac{n \pi}{M}\right)\right|^{2} .
$$


For more details see the paper [26]. In the next result we consider an extrapolation problem, see also [18,29,30,34], solved with the techniques developed in Sect. 4:

Theorem 6.4 Let $s$ be a signal with band-limited spectrum. Then, for $t \in \mathbb{R} \backslash[-1,1]$,

$$
s(t)=\lim _{n \rightarrow \infty} \sum_{k=0}^{n} c_{k}(n, t) s(1-2 k / n) .
$$

Proof Since the sequence of function $\omega \mapsto F_{n}(\omega, t)$ converges uniformly on compact sets to $e^{i t \omega}$ we can write, as done in (4.4)

$$
\int_{K} e^{i a t} d \mu(t)=\lim _{n \rightarrow \infty} \sum_{k=0}^{n} c_{k}(n, a) D(1-2 k / n),
$$

which is (6.10).

Acknowledgements The authors are grateful to the referee for the useful comments.

Funding Open access funding provided by Politecnico di Milano within the CRUI-CARE Agreement.

Open Access This article is licensed under a Creative Commons Attribution 4.0 International License, which permits use, sharing, adaptation, distribution and reproduction in any medium or format, as long as you give appropriate credit to the original author(s) and the source, provide a link to the Creative Commons licence, and indicate if changes were made. The images or other third party material in this article are included in the article's Creative Commons licence, unless indicated otherwise in a credit line to the material. If material is not included in the article's Creative Commons licence and your intended use is not permitted by statutory regulation or exceeds the permitted use, you will need to obtain permission directly from the copyright holder. To view a copy of this licence, visit http://creativecommons.org/licenses/by/4.0/.

\section{References}

1. Aharonov, Y., Vaidman, L.: Properties of a quantum system during the time interval between two measurements. Phys. Rev. A 41, 11-20 (1990)

2. Aharonov, Y., Rohrlich, D.: Quantum Paradoxes: Quantum Theory for the Perplexed. Wiley-VCH Verlag, Weinheim (2005)

3. Aharonov, Y., Albert, D., Vaidman, L.: How the result of a measurement of a component of the spin of a spin-1/2 particle can turn out to be 100. Phys. Rev. Lett. 60, 1351-1354 (1988)

4. Aharonov, Y., Colombo, F., Sabadini, I., Struppa, D.C., Tollaksen, J.: Some mathematical properties of superoscillations. J. Phys. A 44, 365304 (2011)

5. Aharonov, Y., Colombo, F., Nussinov, S., Sabadini, I., Struppa, D.C., Tollaksen, J.: Superoscillation phenomena in $S O(3)$. Proc. R. Soc. A. 468, 3587-3600 (2012)

6. Aharonov, Y., Colombo, F., Sabadini, I., Struppa, D.C., Tollaksen, J.: On the Cauchy problem for the Schrödinger equation with superoscillatory initial data. J. Math. Pures Appl. 99, 165-173 (2013)

7. Aharonov, Y., Colombo, F., Sabadini, I., Struppa, D.C., Tollaksen, J.: Evolution of superoscillatory data. J. Phys. A 47, 205301 (2014)

8. Aharonov, Y., Colombo, F., Sabadini, I., Struppa, D.C., Tollaksen, J.: Superoscillating sequences as solutions of generalized Schrödinger equations. J. Math. Pures Appl. 103, 522-534 (2015)

9. Aharonov, Y., Colombo, F., Sabadini, I., Struppa, D.C., Tollaksen, J.: Superoscillating sequences in several variables. J. Fourier Anal. Appl. 22(4), 751-767 (2016)

10. Aharonov, Y., Colombo, F., Sabadini, I., Struppa, D.C., Tollaksen, J.: The mathematics of superoscillations. Mem. Am. Math. Soc. 247, 1174 (2017) 
11. Aharonov, Y., Colombo, F., Sabadini, I., Struppa, D.C., Tollaksen, J.: Evolution of superoscillatory initial data in several variables in uniform electric field. J. Phys. A 50(18), 185201 (2017)

12. Aharonov, Y., Sabadini, I., Tollaksen, J., Yger, A.: Classes of superoscillating functions. Quantum Stud. Math. Found. 5, 439-454 (2018)

13. Aharonov, Y., Colombo, F., Struppa, D.C., Tollaksen, J.: Schrödinger evolution of superoscillations under different potentials. Quantum Stud. Math. Found. 5, 485-504 (2018)

14. Aizenberg, L.: Carleman's Formulas in Complex Analysis. Theory and Applications, Revised translation of the 1990 Russian original Mathematics and its Applications, vol. 244. Kluwer Academic Publishers Group, Dordrecht (1993)

15. Akhiezer, N.I.: The Classical Moment Problem. Hafner, New-York (1965)

16. Aoki, T., Colombo, F., Sabadini, I., Struppa, D.C.: Continuity theorems for a class of convolution operators and applications to superoscillations. Ann. Mat. Pura Appl. 4 197(5), 1533-1545 (2018)

17. Aoki, T., Colombo, F., Sabadini, I., Struppa, D.C.: Continuity of some operators arising in the theory of superoscillations. Quantum Stud. Math. Found. 5, 463-476 (2018)

18. Baratchart, L., Leblond, J.: Hardy approximation to $L^{p}$ functions on subsets of the circle with $1 \leq$ $p<\infty$. Constr. Approx. 14, 41-56 (1998)

19. Berry, M.V.: Faster than Fourier. In: Anandan, J.S., Safko, J.L. (eds.) Quantum Coherence and Reality, Celebration of the 60th Birthday of Yakir, Aharonov edn, pp. 55-65. World Scientific, Singapore (1994)

20. Berry, M., Dennis, M.R.: Natural superoscillations in monochromatic waves in D dimension. J. Phys. A 42, 022003 (2009)

21. Berry, M.V., Popescu, S.: Evolution of quantum superoscillations, and optical superresolution without evanescent waves. J. Phys. A 39, 6965-6977 (2006)

22. Buniy, R., Colombo, F., Sabadini, I., Struppa, D.C.: Quantum harmonic oscillator with superoscillating initial datum. J. Math. Phys. 55, 113511 (2014)

23. Colombo, F., Gantner, J., Struppa, D.C.: Evolution of superoscillations for Schrödinger equation in uniform magnetic field. J. Math. Phys. 58(9), 092103 (2017)

24. Colombo, F., Gantner, J., Struppa, D.C.: Evolution by Schrödinger equation of Aharonov-Berry superoscillations in centrifugal potential. Proc. R. Soc. A. 475, 20180390 (2019)

25. Colombo, F., Sabadini, I., Struppa, D.C., Yger, A.: Superoscillating sequences and hyperfunctions. Publ. Res. Inst. Math. Sci 55, 665-688 (2019)

26. Colombo, F., Struppa, D.C., Yger, A.: Superoscillating sequences towards approximation in $\mathcal{S}$ or $\mathcal{S}^{\prime}$-type spaces and extrapolation. J. Fourier Anal. Appl. 25(1), 242-266 (2019)

27. de Branges, L.: Hilbert Spaces of Entire Functions. Prentice-Hall Inc., Englewood Cliffs (1968)

28. Dym, H., McKean Jr., H.P.: Application of de Branges spaces of integral functions to the prediction of stationary Gaussian processes. Illinois J. Math. 14, 299-343 (1970)

29. Dym, H., McKean, H.P.: Gaussian Processes. Function Theory and the Inverse Spectral Problem. Academic Press, New York (1976)

30. Gerchberg, R.W.: Super-resolution through error energy reduction. Optica Acta 21(9), 709-720 (1974)

31. Krein, M.G.: On the problem of continuation of helical arcs in Hilbert space. C. R. (Doklady) Acad. Sci. URSS (N.S.) 45, 139-142 (1944)

32. Krein, M.G.: On a method of effective solution of an inverse boundary problem. Doklady Akad. Nauk SSSR (N.S.) 94, 987-990 (1954)

33. Krein, M.G., Langer, H.: Continuation of Hermitian positive definite functions and related questions. Integral Equ. Operator Theory 78(1), 1-69 (2014)

34. Papoulis, A.: A new algorithm in spectral analysis and band-limited extrapolation. IEEE Trans. Circ. Syst. CAS-2 9, 735-742 (1975)

35. Reed, M., Simon, B.: Methods of Modern Mathematical Physics. I. Functional Analysis, 2nd edn. Academic Press Inc. [Harcourt Brace Jovanovich Publishers], New York (1980)

36. von Neumann, J., Schoenberg, I.J.: Fourier integrals and metric geometry. Trans. Am. Math. Soc. 50, 226-251 (1941)

Publisher's Note Springer Nature remains neutral with regard to jurisdictional claims in published maps and institutional affiliations. 